\title{
THE F. AND M. RIESZ THEOREM AND SOME FUNCTION SPACES
}

\author{
Yuji TAKAHASHI \\ (Received March 12, 1986, Revised August 11, 1986)
}

\section{§ 1. Introduction}

In $1916 \mathrm{~F}$. and M. Riesz published the following result : if $\mu$ is a bounded complex Borel measure on the unit circle $\boldsymbol{T}$ such that

$$
\hat{\mu}(n)=\int_{T} e^{-i n \theta} d \mu(\theta)=0 \quad \text { for } n=-1,-2, \ldots,
$$

then $\mu$ is absolutely continuous with respect to the Lebesgue measure on $\boldsymbol{T}$. Some forty years later, Helson and Lowdenslager generalized this theorem to compact Abelian groups with ordered dual ([5]). Since then a number of related results have been obtained under more general settings ([1], [2], [4], [6], [10], [15], [17]).

In his papers [13] and [14] Sarason showed that $H^{\infty}(\boldsymbol{T})+C(\boldsymbol{T})$ is a closed subalgebra of $L^{\infty}(\boldsymbol{T})$, and that $H^{\infty}(\boldsymbol{R})+C_{u}(\boldsymbol{R})$ is a closed subalgebra of $L^{\infty}(\boldsymbol{R})$. Subsequently, Rudin [12] and Yamaguchi [16] investigated spaces of type $H^{\infty}+C$ on locally compact Abelian groups with ordered dual.

Meanwhile, Hewitt, Koshi, and the author recently presented simple proofs for results in [1], [2], and [17] and recognized that the embedding theorem of a locally compact Abelian group into a locally compact divisible Abelian group is useful in dealing with more general subsemigroups instead of orders $([7])$. In the present paper we continue to use the embedding theorem and study the relation between the F. and M. Riesz theorem and spaces of type $H^{\infty}+C$.

In section 2 we describe our notation and state main theorem which gives a generalization of a theorem of Yamaguchi ([16]]). In fact our result supplies more information on the relation between the $\mathrm{F}$. and M. Riesz theorem and spaces of type $H^{\infty}+C$. Some preliminary lemmas are proved in section 3. We give the proof of our main theorem in section 4 .

\section{$\S 2$. Notation and Main Theorem}

Throughout this paper, the symbols $\boldsymbol{Z}, \boldsymbol{Z}_{+}, \boldsymbol{R}, \boldsymbol{R}_{+}$, and $\boldsymbol{T}$ will denote the integers, the nonnegative integers, the real numbers, the nonnegative real numbers, and the circle group respectively and the term "locally compact 
Abelian group" means "locally compact Abelian group satisfying Hausdorff's separation axiom ".

Let $G$ be a locally compact Abelian group and let $\hat{G}$ denote its dual group. A fixed but arbitrary Haar measure on $G$ will be denoted by $m_{G}$. Let $L^{1}(G)$ be the space of all Haar integrable functions on $G$ and $M(G)$ be the Banach algebra of all bounded regular complex Borel measures on $G$ with convolution multiplication and the total variation norm. As usual, we identify the measures in $M(G)$ that are absolutely continuous with respect to $m_{G}$ with elements of $L^{1}(G)$. Given a subset $E$ of $\hat{G}$, we denote by $M_{E}(G)$ the set of all measures in $M(G)$ whose Fourier-Stieltjes transforms vanish on $\hat{G} \backslash E$.

Let $L^{\infty}(G)$ be the Banach algebra of all complex-valued Haar measurable essentially bounded functions on $G$ under pointwise multiplication and the essential supremum norm and let $C_{u}(G)$ be the Banach algebra of all complex-valued bounded uniformly contiunous functions on $G$ under pointwise multiplication and the supremum norm. (For a compact Abelian group $G$, we simply write $C(G)$ in place of $C_{u}(G)$ ).

For an element $x$ of $G, \delta_{x}$ denotes the Dirac measure at $x$. We denote the Fourier-Stieltjes transform of a measure $\mu$ in $M(G)$ by $\hat{\mu}$ and convolution of measures $\mu$ and $\nu$ in $M(G)$ by $\mu * \nu$. For a closed subgroup $H$ of $G$, $H^{\perp}$ means the annihilator of $H$. For $x \in G$ and $\gamma \varepsilon \hat{G}$, we denote by $(x, \gamma)$ the value of $\gamma$ at $x$.

Recall the definition of an ordered group.

Definition 1. Let $G$ be an Abelian group. $G$ is said to be ordered if there exists a subsemigroup $P$ of $G$ such that

$$
P \cup(-P)=G \text { and } P \cap(-P)=\{0\} \text {. }
$$

For brevity's sake, we will refer to $P$ as an order in $G$.

For example, $\boldsymbol{Z}_{+}$and $\boldsymbol{R}_{+}$are order in $\boldsymbol{Z}$ and $\boldsymbol{R}$ respectively. It is well known that an Abelian group $G$ is ordered if and only if $G$ is torsion-free $([6])$. Details on orders in locally compact Abelian groups can be seen in [6].

The following definition is convenient for our purpose.

DeFinition 2. Let $G$ be a locally compact Abelian group with dual group $\hat{G}$. A subset $E$ in $\hat{G}$ is said to have the $\mathscr{F} \mathscr{R}$ property if $M_{E}(G) \subset$ $L^{1}(G)$.

The F. and M. Riesz theorem says that $\boldsymbol{Z}_{+}$in $\boldsymbol{Z}$ has the FMR property. We can also see that $\boldsymbol{R}_{+}$in $\boldsymbol{R}$ has the FMR property by the F. and M. Riesz 
theorem for $\boldsymbol{R}$ ([11, Theorem 8.2.7]). A well-known theorem of Bochner says that $\boldsymbol{Z}_{+} \times \boldsymbol{Z}_{+}$in $\boldsymbol{Z}^{2}$ has the FMR property (cf. [11, Theorem 8.2.5]).

Now recall a result on the $F$. and $M$. Riesz theorem and group structures. It is due to Hewitt, Koshi, and Yamaguchi.

TheOREM A ([6] and [10]). Let $G$ be a locally compact Abelian group with ordered dual group $\hat{G}$ and let $P$ be an order in $\hat{G}$ which is not dense in $\hat{G}$. Then $P$ has the FMR property if and only if $G$ is isomorphic to $\boldsymbol{R} \times \Delta$ or $\boldsymbol{T} \times \Delta$, where $\Delta$ is discrete. Moreover, there exists $\mu \varepsilon M_{P}(G)$ which is singular with respect to $m_{G}$ unless $G$ is isomorphic to $\boldsymbol{R} \times \Delta$ or $\boldsymbol{T} \times \Delta$ for a discrete group $\Delta$.

We give another definition to state our main theorem.

Definition 3. Let $G$ be a locally compact Abelian group with dual group $\hat{G}$ and let $P$ be a subsemigroup of $\hat{G}$ such that $P \cup(-P)=\hat{G}$. We define $H_{P}^{1}(G)$ and $H_{P}^{\infty}(G)$ as follows:

$$
\begin{aligned}
& H_{P}^{1}(G)=\left\{f \varepsilon L^{1}(G): \hat{f}(\gamma)=0 \text { for } \gamma \text { \& } P\right\} ; \\
& H_{P}^{\infty}(G)=\left\{g \varepsilon L^{\infty}(G): \int_{G} f(x) g(x) d m_{G}(x)=0 \text { for } f \varepsilon H_{P}^{1}(G)\right\} .
\end{aligned}
$$

Let $H^{1}(\boldsymbol{R}), H^{\infty}(\boldsymbol{R}), H^{1}(\boldsymbol{T})$ and $H^{\infty}(\boldsymbol{T})$ denote the usual Hardy spaces. If $G=\boldsymbol{R}$ and $P=\boldsymbol{R}_{+}$, then $H_{P}^{1}(G)=H^{1}(\boldsymbol{R})$ and $H_{P}^{\infty}(G)=H^{\infty}(\boldsymbol{R})$. If $G=\boldsymbol{T}$ and $P=\boldsymbol{Z}_{+}$, then $H_{P}^{1}(G)=H^{1}(\boldsymbol{T})$ and $H_{P}^{\infty}(G)=H_{0}^{\infty}(\boldsymbol{T})$, where $H_{0}^{\infty}(\boldsymbol{T})=\{f$ $\left.\varepsilon H^{\infty}(\boldsymbol{T}): \hat{f}(0)=0\right\}$. Note that $H_{0}^{\infty}(\boldsymbol{T})+\mathrm{C}(\boldsymbol{T})=H^{\infty}(\boldsymbol{T})+C(\boldsymbol{T}) . \quad H^{\infty}(\boldsymbol{T})+$ $C(\boldsymbol{T})$ and $H^{\infty}(\boldsymbol{R})+C_{u}(\boldsymbol{R})$ are a closed subalgebra of $L^{\infty}(\boldsymbol{T})$ and a closed subalgebra of $L^{\infty}(\boldsymbol{R})$ respectively ([13], [14]).

Yamaguchi showed the following theorem, which generalized an earlier result of Rudin.

Theorem B ([16]). Let $G$ be a locally compact Abelian group with ordered dual group $\hat{G}$ and let $P$ be an order in $\hat{G}$ which is not dense in $\hat{G}$. Then $H_{P}^{\infty}(G)+C_{u}(G)$ is an algebra if and only if $G$ is isomorphic to $\boldsymbol{R} \times \Delta$ or $\boldsymbol{T} \times \Delta$, where $\Delta$ is discrete.

Rudin ([12, Theorem 3.6]) proved Theorem B for the case where $G$ is compact.

From Theorems A and B we can see that an order $P$ in $\hat{G}$ which is not dense in $\hat{G}$ has the FMR property if and only if $H_{P}^{\infty}(G)+C_{u}(G)$ is an algebra. Our purpose in this paper is to prove this equivalence under a more general setting that $P$ is a subsemigroup in $\hat{G}$ such that $P \cup(-P)=\hat{G}$. Our treatment seems to supply directer and simpler one to relate two properties. 
The key to our arguments is to connect these two properties through certain conditions concerning measures.

We now state our main theorem.

THEOREM. Let $G$ be a locally compact Abelian group with dual group $\hat{G}$ and let $P$ be a subsemigroup of $\hat{G}$ which is not dense in $\hat{G}$ such that $P \cup(-P)=$ $\hat{G}$. Then the following statements are equivalent.

(i) $H_{P}^{\infty}(G)+C_{u}(G)$ is an algebra.

(ii) $\mu *(\gamma \nu) \varepsilon L^{1}(G)$ for each $\mu \varepsilon M_{P^{c}}(G), \nu \varepsilon M_{(-P)^{c}}(G)$, and $\gamma \varepsilon$ $\hat{G}$.

(iii) $\mu *(\gamma \nu) \varepsilon L^{1}(G)$ for each $\mu$ and $\nu \varepsilon M_{P^{c}}(G)$ and $\gamma \varepsilon \hat{G}$.

(iv) $P^{c}$ has the FMR property.

(v) $P$ has the FMR property.

(vi) $G$ is isomorphic to $\boldsymbol{R} \times \Delta$ or $\boldsymbol{T} \times \Delta$, where $\Delta$ is discrete.

REMARK $1 . \quad$ It is easy to see that $H_{P}^{\infty}(G)$ is a weak*-closed translation invariant subspace of $L^{\infty}(G)$. Hence $H_{P}^{\infty}(G)+C_{u}(G)$ is a closed subspace of $L^{\infty}(G)$ by [12, Theorem 3.3].

Finally recall the embedding theorem of locally compact Abelian groups mentioned in section 1 .

Theorem C ([8, (25. 32) (a) and Theorem (A. 15)]). Let $G$ be a locally compact Abelian group. Then there exists a locally compact divisible Abelian group $G_{0}$ which contains $G$ as an open subgroup.

Note that $\hat{G}_{0}$ is torsion-free ([9, Theorem (24.23)]) and therefore $\hat{G}_{0}$ is ordered.

\section{$\S 3$. Some Lemmas}

The following fact is easily seen; so we omit the proof.

Lemma 1. Let $G$ be a torsion-free Abelian group and let $S$ be a subsemigroup of $G$ such that $S \cup(-S)=G$. Then $S \cap(-S)$ has an order and, for any such order $P$, the set $(S \backslash(-S)) \cap P$ is an order in $G$.

Lemma 2. Let $G$ be a locally compact Abelian group and let $\mu$ be an element of $M(G)$. Then $\mu \in L^{1}(G)$ if and only if $\mu * L^{\infty}(G) \subset C_{u}(G)$.

Proof. We can see that $\mu \varepsilon L^{1}(G)$ implies $\mu * L^{\infty}(G) \subset C_{u}(G)$ by [8, Theorem (20.16)]. Conversely, suppose $\mu * L^{\infty}(G) \subset C_{u}(G)$. Then we in particular have

$$
\mu(x-E)=\mu * \chi_{E}(x) \varepsilon C_{u}(G)
$$


for any Borel subset $E$ of $G$, where $\chi_{E}$ denotes the characteristic function of $E$. By $[8,(19.27)]$, we have $\mu \in L^{1}(G)$.

Lemma 3. Let $G$ be a locally compact Abelian group and let $P$ be a subsemigroup of $\hat{G}$ satisfying $P \cup(-P)=\hat{G}$.

(a) If $f \varepsilon L^{\infty}(G)$ and $\mu \varepsilon M_{(-P)^{c}}(G)$, then $f * \mu \varepsilon H_{P}^{\infty}(G)$.

(b) If $h \in H_{P}^{\infty}(G)$ and $\nu \in M_{(-P)}(G)$, then $h * \nu=0$.

Proof. (a) For $g \in H_{P}^{1}(G)$, we define $\tilde{g}(x)=g(-x)$. Then, since $\hat{\tilde{g}}(\gamma)=\hat{g}(-\gamma)=0$ for all $\gamma d-P, \hat{\mu} \cdot \hat{\tilde{g}} \equiv 0$ and so $\mu * \tilde{g}=0$. Hence we have

$$
\begin{aligned}
\int_{G}(f * \mu)(x) g(x) d m_{G}(x) & =\int_{G}(f * \mu)(x) \tilde{g}(-x) d m_{G}(x) \\
& =((f * \mu) * \tilde{g})(0) \\
& =(f *(\mu * \tilde{g}))(0) \\
& =0 .
\end{aligned}
$$

This implies $f * \mu \varepsilon H_{P}^{\infty}(G)$.

(b) Let $k \varepsilon L^{1}(G)$. As in (a) we consider the function $\tilde{k}$ defined by $\tilde{k}(x)=k(-x)$. Then we have

$$
\begin{aligned}
\int_{G}(h * \nu)(x) k(x) d m_{G}(x) & =\int_{G}(h * \nu)(x) \tilde{k}(-x) d m_{G}(x) \\
& =((h * \nu) * \tilde{k})(0) \\
& =(h *(\nu * \tilde{k}))(0) \\
& =\int_{G} h(x)(\boldsymbol{\nu} * \tilde{k})(-x) d m_{G}(x) \\
& =\int_{G} h(x)(\boldsymbol{\nu} * \tilde{k})^{\tilde{C}}(x) d m_{G}(x) .
\end{aligned}
$$

Since

$$
((\nu * \tilde{k}) \tilde{)} \hat{)}(\gamma)=\hat{\nu}(-\gamma) \hat{k}(\gamma)=0
$$

for all $\gamma$ \& $P$, we have $(\nu * \tilde{k})^{\sim} \varepsilon H_{P}^{1}(G)$. This implies

$$
\int_{G}(h * \nu)(x) k(x) d m_{G}(x)=0 .
$$

Since $k$ is an arbitrary element of $L^{1}(G)$, we have $h * \nu=0$.

Lemma 4. Let $G$ be a locally compact Abelian group and let $\mu$ and $\nu$ be elements of $M(G)$. Then $\left(\gamma_{1} \mu\right) *\left(\gamma_{2} \nu\right) \varepsilon L^{1}(G)$ for all $\gamma_{1}$ and $\gamma_{2} \varepsilon \hat{G}$ if and only if $|\mu| *|\nu| \varepsilon L^{1}(G)$, where $|\mu|$ denotes the total variation measure of $\mu$. 
Proof. Suppose $|\boldsymbol{\mu}| *|\nu| \varepsilon L^{1}(G)$. If $A$ is a subset of $G$ with $m_{G}(A)=$ 0 , then $(|\mu| *|\nu|)(A)=0$ and therefore

$$
\left|\left(\left(\gamma_{1} \mu\right) *\left(\gamma_{2} \nu\right)\right)(A)\right| \leqq\left(\left|\gamma_{1} \mu\right| *\left|\gamma_{2} \nu\right|\right)(A)=(|\mu| *|\nu|)(A)=0 .
$$

Hence, $\left(\gamma_{1} \mu\right) *\left(\gamma_{2} \nu\right)$ belongs to $L^{1}(G)$.

Conversely, suppose $\left(\gamma_{1} \mu\right) *\left(\gamma_{2} \nu\right) \varepsilon \mathrm{L}^{1}(\mathrm{G})$ for all $\gamma_{1}$ and $\gamma_{2} \varepsilon \hat{G}$. Then we have $\left(p_{1} \mu\right) *\left(p_{2} \nu\right) \varepsilon L^{1}(G)$ for all trigonometric polynomials $p_{1}$ and $p_{2}$ on $G$. Now choose sequences $\left\{p_{n}\right\}$ and $\left\{q_{n}\right\}$ of trigonometric polynomials on $G$ such that

$$
\lim _{n \rightarrow \infty}\left\|p_{n} \mu-|\mu|\right\|=0 \text { and } \lim _{n \rightarrow \infty}\left\|q_{n} \nu-|\nu|\right\|=0 .
$$

(Note that the set of all trigonometric polynomials is dense in $L^{1}(|\mu|)$, where $L^{1}(|\mu|)$ denotes $L^{1}$-space with respect to $|\mu|$.) Then we have

$$
\lim _{n \rightarrow \infty}\left\|\left(p_{n} \mu\right) *\left(q_{n} \nu\right)-|\mu| *|\nu|\right\|=0 .
$$

Since $\left(p_{n} \mu\right) *\left(q_{n} \nu\right) \varepsilon L^{1}(G)$ for all $n$ and $L^{1}(G)$ is a closed subspace of $M$ $(G)$, we have $|\boldsymbol{\mu}| *|\nu| \varepsilon L^{1}(G)$.

Lemma 5. Let $G=\boldsymbol{R} \times K$ for a compact Abelian group $K$ and let $P$ be a subsemigroup of $G$ which is not dense in $G$ such that $P \cup(-P)=G$. Then $P$ satisfies the following (a) or (b):

(a) $P=\{(r, k) \varepsilon \boldsymbol{R} \times K: r>0$ and $k \varepsilon K\} \cup(P \cap(\{0\} \times K))$;

(b) $P=\{(r, k) \varepsilon \boldsymbol{R} \times K: r<0$ and $k \varepsilon K\} \cup(P \cap(\{0\} \times K))$.

Proof. Let $\pi$ denote the projection from $G$ onto $\boldsymbol{R}$. Then $\pi$ is a closed mapping because $K$ is compact ([8, Theorem (5.18)]). Hence $\tilde{P}=\pi(\bar{P})$ is a closed subsemigroup of $\boldsymbol{R}$, where $\bar{P}$ denotes the closure of $P$. Moreover, $\tilde{P}$ is proper in $\boldsymbol{R}$. Indeed, choose $x \in \bar{P}$ such that $-x \& \bar{P}$. Then, noting that a closed subsemigroup of a compact group is a subgroup ([8, Theorem (9.16) $)$, we have $-x$ \& $\bar{P}+(\{0\} \times K)$. If $\tilde{P}=\boldsymbol{R}$, then

$$
\boldsymbol{R} \times K=\pi^{-1}(\tilde{P})=\bar{P}+(\{0\} \times K) .
$$

But this is a contradiction because $-x \notin \bar{P}+(\{0\} \times K)$. Thus $\tilde{P}$ is a proper closed subsemigroup of $\boldsymbol{R}$ satisfying $\tilde{P} \cup(-\tilde{P})=\boldsymbol{R}$. Now suppose that $\tilde{P}$ contains both a positive number and a negative one. Then the following three cases are considered: (a) $\tilde{P}$ has both a positive minimum element and a negative maximum one; (b) $\tilde{P}$ has neither a positive minimum element nor a negative maximum one; (c) neither (a) nor (b) holds. Clearly (c) is impossible. If (a) holds, then it is easy to see that $\tilde{P}=c \boldsymbol{Z}$ for $c=$ 
$\min \{\tilde{P} \cap(\boldsymbol{R} \backslash\{0\})\}$ but this is a contradiction because $\tilde{P} \cup(-\tilde{P})=\boldsymbol{R}$. If (b) holds, then we can easily show that $\tilde{P}$ is dense in $\boldsymbol{R}$. Since $\tilde{P}$ is closed, we have $\tilde{P}=\boldsymbol{R}$. But this is a contradiction because $\tilde{P}$ is proper in $\boldsymbol{R}$. Thus we saw that $\tilde{P} \subset[0,+\infty)$ or $\tilde{P} \subset(-\infty, 0]$. But since $\tilde{P} \cup(-\tilde{P})=\boldsymbol{R}$, we have

$$
\tilde{P}=[0,+\infty) \text { or }(-\infty, 0] \text {. }
$$

Let $\tilde{P}=[0,+\infty)$. Then it is clear that

$$
P \subset\{(r, k) \varepsilon \boldsymbol{R} \times K: r \geqq 0 \text { and } k \varepsilon K\} .
$$

Let $r>0$ and $k \varepsilon K$. If $(r, k)$ d $P$, then $(r, k) \varepsilon-P$, and so $(-r,-k) \varepsilon$ $P$. But this contradicts $\tilde{P}=[0,+\infty)$. Thus we have (a). If $\tilde{P}=(-\infty, 0]$, we can show (b) by the same argument.

REMARK 2. By an argument similar to the one used above, we can prove the following. Let $G=\boldsymbol{Z} \times K$ for a compact Abelian group $K$ and let $P$ be a subsemigroup of $G$ which is not dense in $G$ such that $P \cup(-P)=G$. Then $P$ satisfies the following (a) or (b):

(a) $P=\{(n, k) \varepsilon \boldsymbol{Z} \times K: n>0$ and $k \varepsilon K\} \cup(P \cap(\{0\} \times K))$;

(b) $P=\{(n, k) \varepsilon \boldsymbol{Z} \times K: n<0$ and $k \varepsilon K\} \cup(P \cap(\{0\} \times K))$.

The following lemma holds under a more general setting; see [9].

LEMMA 6. Let $G$ be a torsion-free locally compact Abelian group and let $P$ be a subsemigroup of $G$ which is not dense in $G$ such that $P \cup(-P)=G$. Then there exist an element $x_{0}$ in $G$ and an order $P_{0}$ in $G$ which is not dense in $G$ such that $x_{0}+P$ is included in $P_{0}$.

Proof. If $P \cap(-P)=\{0\}$, then we have only to put $P_{0}=P$. Let $P \cap$ $(-P) \neq\{0\}$. Then, by Lemma 1, there exists an order $P_{0}$ in $G$ such that

$$
P \backslash(-P) \subset P_{0} \subset P \text {. }
$$

Of course $P_{0}$ is not dense in $G$ because $P$ is so. Choose and fix any $x_{0} \varepsilon P \backslash$ $(-P)$. Then we can easily show that $P_{0}$ contains $x_{0}+P$.

LemMA 7. Let $G$ be a locally compact Abelian group and let $H$ be an open subgroup of $G$. Let $\tilde{E}$ be a subset of $\hat{G} / H^{\perp}$ and put $E=\pi^{-1}(\tilde{E})$, where $\pi$ denotes the natural homomorphism from $\hat{G}$ onto $\hat{G} / H^{\perp}$. If $\mu \varepsilon M_{E}(G)$, then $\left(\mu_{x+H}\right) * \delta_{-x} \varepsilon M_{\tilde{E}}(H)$ for all $x \in G$, where $\mu_{x+H}$ denotes the restriction of $\mu$ to the coset $x+H$.

PROOF. The proof of this lemma is based on the argument in [15, pp. 114-115]. Since $\mu$ has $\sigma$-compact support, there exists a sequence $\left\{x_{n}\right\}$ consisting of elements in $G$ such that 


$$
\mu=\sum_{n=1}^{\infty} \mu_{x_{n}+H} \text { and } x_{i}+H \neq x_{j}+H \quad(i \neq j) .
$$

Note that

$$
\|\mu\|=\sum_{n=1}^{\infty}\left\|\mu_{x_{n}+H}\right\| .
$$

We have only to show that

$$
\left(\mu_{x_{n}+H}\right) * \delta_{-x_{n}} \varepsilon M_{\tilde{E}}(H)
$$

for all $n$. We first claim that

$$
\mu_{x_{n}+H} \varepsilon M_{E}(G)
$$

for all $n$. To show this claim, let $f$ be a function in $L^{1}(\hat{G})$ with $\operatorname{supp}(f) \subset$ $E^{c}$, where $\operatorname{supp}(f)$ denotes the support of $f$. Then we have

$$
\begin{aligned}
0 & =\int_{G} \hat{\mu}(\gamma) f(\boldsymbol{\gamma}) d m_{\hat{G}}(\gamma) \\
& =\int_{G} \hat{f}(x) d \mu(x) \\
& =\sum_{n=1}^{\infty}\left(\mu_{x_{n}+H}\right)(\hat{f}) .
\end{aligned}
$$

For $\gamma_{*} \varepsilon H^{\perp}$, we define $f_{\gamma_{*}} \varepsilon L^{1}(\hat{G})$ by $f_{\gamma_{*}}(\gamma)=f\left(\gamma-\gamma_{*}\right)$. Then, since $H^{\perp}+$ $E^{c} \subset E^{\mathrm{c}}$, we have $\operatorname{supp}\left(f_{\gamma_{*}}\right) \subset E^{c}$. Hence

$$
\begin{aligned}
0 & =\sum_{n=1}^{\infty}\left(\mu_{x_{n}+H}\right)\left(\left(f_{\gamma_{*}}\right)^{\hat{)}}\right) \\
& =\sum_{n=1}^{\infty} \int_{G}\left(-x, \gamma_{*}\right) \hat{f}(x) d\left(\mu_{x_{n}+H}\right)(x) \\
& =\sum_{n=1}^{\infty}\left(-\dot{x}_{n}, \gamma_{*}\right) \int_{G} \hat{f}(x) d\left(\mu_{x_{n}+H}\right)(x) \\
& =\sum_{n=1}^{\infty}\left(-\dot{x}_{n}, \gamma_{*}\right)\left(\mu_{x_{n}+H}\right)(\hat{f}),
\end{aligned}
$$

where $x_{n} \varepsilon \dot{x}_{n} \varepsilon G / H$. Since $\gamma$, is an arbitrary element in $H^{\perp}$, we have

$$
\begin{aligned}
0 & =\sum_{n=1}^{\infty} p\left(\dot{x}_{n}\right)\left(\mu_{x_{n}+H}\right)(\hat{f}) \\
& =\int_{G / H} p(\dot{x}) \mu_{\dot{x}}(\hat{f}) d m_{G / H}(\dot{x})
\end{aligned}
$$

for all trigonometric polynomials $p$ on $G / H$, where $\mu_{\dot{x}}(\hat{f})=\left(\mu_{x+H}\right)(\hat{f})$ with $x \in \dot{x}$. Since the function $\dot{x} \longrightarrow \mu_{\dot{x}}(\hat{f})$ is an element of $L^{1}(G / H)$ and the set 
of all trigonometric polynomials on $G / H$ is weak ${ }^{*}$-dense in $L^{\infty}(G / H)$, we have $\left(\mu_{x_{n}+H}\right)(\hat{f})=0$ for all $n$. Hence

$$
\begin{aligned}
\int_{G}\left(\mu_{x_{n}+H}\right) \hat{\wedge}(\gamma) f(\gamma) d m_{\hat{G}}(\gamma) & =\int_{G} \hat{f}(x) d\left(\mu_{x_{n}+H}\right)(x) \\
& =\left(\mu_{x_{n}+H}\right)(\hat{f}) \\
& =0
\end{aligned}
$$

for all $n$. Since $f$ is an arbitrary function in $L^{1}(\hat{G})$ with $\operatorname{supp}(f) \subset E^{c}$, we have

$$
\mu_{x_{n}+H} \varepsilon M_{E}(G)
$$

for all $n$. Thus we showed the claim. Now recall that the dual group of $H$ is $\hat{G} / H^{\perp}$. As a measure in $M(H)$, each $\left(\mu_{x_{n}+H}\right) * \delta_{-x_{n}}$ has a Fourier-Stieltjes transform constant on cosets of $H^{\perp}$. Thus we may write $\left(\left(\mu_{x_{n}+H}\right) * \delta_{-x_{n}}\right)^{\wedge}$ $\left(\gamma+H^{\perp}\right)$ for $\gamma \varepsilon \hat{G}$. For $\gamma+H^{\perp}$ with $\gamma \varepsilon E^{c}$, we have

$$
\begin{aligned}
\left(\left(\mu_{x_{n}+H}\right) * \delta_{-x_{n}}\right) \hat{\wedge}\left(\gamma+H^{\perp}\right) & =\int_{H}\left(-h, \gamma+H^{\perp}\right) d\left(\left(\mu_{x_{n}+H}\right) * \delta_{-x_{n}}\right)(h) \\
& =\int_{G}(-z, \gamma) d\left(\left(\mu_{x_{n}+H}\right) * \delta_{-x_{n}}\right)(z) \\
& =\int_{G} \int_{G}(-x-y, \gamma) d\left(\mu_{x_{n}+H}\right)(x) d \delta_{-x_{n}}(y) \\
& =\left(x_{n}, \gamma\right) \int_{G}(-x, \gamma) d\left(\mu_{x_{n}+H}\right)(x) \\
& =\left(x_{n}, \gamma\right)\left(\mu_{x_{n}+H}\right)^{\wedge}(\gamma) \\
& =0 .
\end{aligned}
$$

Hence we have

$$
\left(\mu_{x_{n}+H}\right) * \delta_{-x_{n}} \varepsilon M_{\hat{E}}(H)
$$

for all $n$.

\section{$\S 4$. Proof of Main Theorem}

( i ) $\Rightarrow$ (ii): Suppose that there exist $\mu \varepsilon M_{P^{c}}(G), \nu \varepsilon M_{(-P)^{c}}(G)$, and $\gamma_{0}$ $\varepsilon \hat{G}$ such that $\mu *\left(\gamma_{0} \nu\right)$ \& $L^{1}(G)$. Then, by Lemma 2, there exists $f \varepsilon L^{\infty}(G)$ such that

$$
f *\left(\mu *\left(\gamma_{0} \nu\right)\right) \text { \&े } C_{u}(G) \text {. }
$$

Since $\nu \in M_{(-P)^{c}}(G)$, we have $\left(\bar{\gamma}_{0} f\right) * \nu \varepsilon H_{P}^{\infty}(G)$ by Lemma 3 (a). We claim that

$$
\gamma_{0} \cdot\left(\left(\overline{\gamma_{0}} f\right) * \nu\right) \text { dे } H_{P}^{\infty}(G)+C_{u}(G) .
$$


Since $\gamma_{0} \varepsilon C_{u}(G)$ and $\left(\bar{\gamma}_{0} f\right) * \nu \varepsilon H_{P}^{\infty}(G)$, we obtain that $H_{P}^{\infty}(G)+C_{u}(G)$ is not an algebra. To prove the claim, suppose

$$
\gamma_{0} \bullet\left(\left(\bar{\gamma}_{0} f\right) * v\right)=g+h
$$

for some $g \varepsilon C_{u}(G)$ and $h \varepsilon H_{P}^{\infty}(G)$. Then we have

$$
\left(\gamma_{0} \cdot\left(\left(\bar{\gamma}_{0} f\right) * \nu\right)\right) * \mu=g * \mu+h * \mu .
$$

Since $h \varepsilon H_{P}^{\infty}(G)$ and $\mu \varepsilon M_{P^{c}}(G) \subset M_{(-P)}(G)$, we have $h * \mu=0$ by Lemma 3 (b). Since $g \in C_{u}(G)$ implies $g * \mu \varepsilon C_{u}(G)$,

$$
f *\left(\mu *\left(\gamma_{0} \nu\right)\right)=\left(f *\left(\gamma_{0} \boldsymbol{\nu}\right)\right) * \mu=\left(\gamma_{0} \bullet\left(\left(\bar{\gamma}_{0} f\right) * \nu\right)\right) * \mu \quad \varepsilon \quad C_{u}(G) .
$$

But this is a contradiction.

(ii ) (iii): For $\sigma \varepsilon M(G)$, we define elements $\sigma^{\prime}$ and $\tilde{\sigma}$ as follows :

$$
\sigma^{\prime}(E)=\sigma(-E) \text { and } \tilde{\sigma}(E)=\overline{\sigma(-E)}
$$

for any Borel subset $E$ of $G$. Then it is easy to see that

$$
\left(\sigma^{\wedge}\right)^{\wedge}(\gamma)=\hat{\sigma}(-\gamma),(\tilde{\sigma})^{\wedge}(\gamma)=\overline{\hat{\sigma}(\gamma)},
$$

and therefore

$$
((\tilde{\sigma}))^{\wedge}(\gamma)=\overline{\hat{\sigma}(-\gamma)}
$$

for any $\gamma \in \hat{G}$. Also note that

$$
|\sigma|^{\sim}=|\tilde{\sigma}|=\left|\sigma^{\prime}\right|=|\sigma|^{\prime},\left(|\sigma|^{\sim}\right)^{\prime}=|\sigma|,
$$

and so we have $\left|(\tilde{\sigma})^{\prime}\right|=|\sigma|$. Thus we obtain that $\sigma \varepsilon M_{(-P)^{c}}(G)$ if and only if $(\tilde{\sigma})^{\prime} \varepsilon M_{P^{c}}(G)$. Suppose that $\mu *(\gamma \nu) \varepsilon L^{1}(G)$ for all $\mu \varepsilon M_{P^{c}}(G)$, $\nu \varepsilon M_{(-P)^{c}}(G)$, and $\gamma \varepsilon \hat{G}$. Then

$$
\left(\gamma_{1} \mu\right) *\left(\gamma_{2} \nu\right)=\gamma_{1} \cdot\left(\mu *\left(\bar{\gamma}_{1} \gamma_{2} \nu\right)\right) \varepsilon L^{1}(G)
$$

for all $\mu \varepsilon M_{P^{c}}(G), \nu \varepsilon M_{(-P)^{c}}(G)$, and $\gamma_{1}$ and $\gamma_{2} \varepsilon \hat{G}$. Hence, by Lemma 4 , we have

$$
|\mu| *|\nu| \varepsilon L^{1}(G) \text { and so }|\mu| *\left|(\tilde{\boldsymbol{\nu}})^{\prime}\right| \varepsilon L^{1}(G)
$$

for all $\mu \varepsilon M_{P^{c}}(G)$ and $\nu \varepsilon M_{(-P)^{c}}(G)$. Again, by Lemma 4, we have

$$
\mu *(\gamma(\tilde{\boldsymbol{\nu}})) \varepsilon L^{1}(G)
$$

for all $\mu \varepsilon M_{P^{c}}(G), \nu \varepsilon M_{(-P)^{c}}(G)$, and $\gamma \varepsilon \hat{G}$. Since $\sigma \varepsilon M_{(-P)^{c}}(G)$ if and only if $(\tilde{\sigma})^{\prime} \varepsilon M_{P^{c}}(G)$, we have 


$$
\mu *(\gamma \nu) \varepsilon L^{1}(G)
$$

for all $\mu$ and $\nu \varepsilon M_{P^{c}}(G)$ and $\gamma \varepsilon \hat{G}$. Thus (ii) implies (iii). To prove that (iii) implies (ii), we have only to trace this argument conversely.

(iii) $\Rightarrow(v i)$ : By Theorem $\mathrm{C}$, there exists a locally compact divisible Abelian group $G_{0}$ such that $G$ is an open subgroup of $G_{0}$. Then $\hat{G}_{0}$ is torsion-free. Suppose that $G$ is neither of the form $\boldsymbol{R} \times \Delta$ nor $\boldsymbol{T} \times \Delta$ for a discrete group $\Delta$. Since an open subgroup of $\boldsymbol{R} \times \Delta(\boldsymbol{T} \times \Delta)$ has the form of $\boldsymbol{R} \times \Delta^{\prime}$ (resp. $\boldsymbol{T} \times \Delta^{\prime}$ ) for a subgroup $\Delta^{\prime}$ of $\Delta$, we see that $G_{0}$ is neither of the form $\boldsymbol{R} \times \Delta$ nor $\boldsymbol{T} \times \Delta$ for a discrete group $\Delta$. Put

$$
P_{0}=\pi^{-1}(P) \text {, }
$$

where $\pi$ denotes the natural homomorphism from $\hat{G}_{0}$ onto $\hat{G}_{0} / G^{\perp}$. (Recall that the dual group of $G$ is $\hat{G}_{0} / G^{\perp}$.) Then $P_{0}$ is a subsemigroup of $\hat{G}_{0}$ which is not dense in $\hat{G}_{0}$ such that $P_{0} \cup\left(-P_{0}\right)=\hat{G}_{0}$. By Lemma 6, there exist $\gamma_{0} \varepsilon$ $\hat{G}_{0}$ and an order $P_{1}$ in $\hat{G}_{0}$ which is not dense in $\hat{G}_{0}$ such that $P_{1}$ contains $\gamma_{0}+$ $P_{0}$. Since $G_{0}$ is neither of the form $\boldsymbol{R} \times \Delta$ nor $\boldsymbol{T} \times \Delta$ for a discrete group $\Delta$, we can find $\mu \varepsilon M_{P_{1}}\left(G_{0}\right)$ such that $\hat{\mu}$ \& $C_{0}\left(\hat{G}_{0}\right)$, where $C_{0}\left(\hat{G}_{0}\right)$ denotes the space of all continuous functions on $\hat{G}_{0}$ which vanish at infinity $([10])$. Of course this measure $\mu$ is included in $M_{\left(\gamma_{0}+P_{0}\right)}\left(G_{0}\right)$. Now consider the measure $\nu=$ $\bar{\gamma}_{0} \mu \varepsilon M\left(G_{0}\right)$. Then $\nu$ is a measure in $M_{P_{0}}\left(G_{0}\right)$ such that $\hat{\nu}$ \& $C_{0}\left(\hat{G}_{0}\right)$ and therefore $\nu * \nu$ \& $L^{1}\left(G_{0}\right)$. Since $\nu$ has $\sigma$-compact support and $G$ is an open subgroup of $G_{0}$, there exists a sequence $\left\{x_{n}\right\}$ of elements of $G_{0}$ such that

$$
\nu=\sum_{n=1}^{\infty} \nu_{x_{n}+G} \text { and } x_{i}+G \neq x_{j}+G(i \neq j) .
$$

Note that the above series converges to $\nu$ in the total variation norm. Put

$$
\nu_{n}=\nu_{x_{n}+G} * \delta_{-x_{n}}
$$

for $n=1,2, \cdots$. Then by Lemma 7 we have

$$
\nu_{n} \varepsilon M_{P^{c}}(G)
$$

for $n=1,2, \cdots$. Since

$$
\nu * \nu=\sum_{m, n=1}^{\infty} \nu_{m} * \nu_{n} * \delta_{x_{m}} * \delta_{x_{n}}
$$

and this series converges in the total variation norm, we can find $m_{0}$ and $n_{0}$ such that

$$
\nu_{m_{0}} * \nu_{n_{0}} * \delta_{x_{0}} * \delta_{x_{n_{0}}} \text { वे } L^{1}\left(G_{0}\right) .
$$


Thus $\nu_{m_{0}}{ }^{*} \nu_{n_{0}}$ is a measure in $M(G)$ such that $\nu_{m_{0}}{ }^{*} \nu_{n_{0}}$ \& $L^{1}\left(G_{0}\right)$. Since $G$ is an open subgroup of $G_{0}$, we have $\boldsymbol{\nu}_{m_{0}} \boldsymbol{\nu}_{n_{0}}$ \& $L^{1}(G)$. Thus (iii) implies (vi).

(vi) $\Rightarrow$ (iv): The proof of this implication is included in $[4$, section 2]. But his proof seems to be obscure. A very simple proof can be seen in [9].

(iv) $\Rightarrow$ (vi): This implication has been already proved in the implication “ (iii) $\Rightarrow(\mathrm{vi})$ ”.

(vi) $\Rightarrow$ ( i ) : Suppose $G$ is isomorphic to $\boldsymbol{R} \times \Delta$ or $\boldsymbol{T} \times \Delta$ for a discrete group $\Delta$. Let $K$ be the dual group of $\Delta$. Thus $K$ is compact. We first consider the case where $G$ is isomorphic to $\boldsymbol{R} \times \Delta$. By Lemma 5 , we may suppose

$$
P=\{(r, k) \varepsilon \boldsymbol{R} \times K: r>0 \text { and } k \varepsilon K\} \cup(P \cap(\{0\} \times K)) .
$$

We claim that

$$
H_{P}^{1}(\boldsymbol{R} \times \Delta)=\left\{f \varepsilon L^{1}(\boldsymbol{R} \times \Delta): f(\bullet, d) \varepsilon H^{1}(\boldsymbol{R}) \text { for each } d \varepsilon \Delta\right\} .
$$

To show this claim, let $r \varepsilon \boldsymbol{R}$ with $r<0$ and let $f \varepsilon H_{P}^{1}(\boldsymbol{R} \times \Delta)$. From the form of $P$ we have $(r, k)$ \& $P$ for each $k \varepsilon K$. Thus

$$
\begin{aligned}
& \int_{\Delta}(-d, k) \int_{\boldsymbol{R}} e^{-i x r} f(x, d) d m_{\boldsymbol{R}}(x) d m_{\Delta}(d) \\
& =\int_{\Delta} \int_{\boldsymbol{R}}(-(x, d),(r, k)) f(x, d) d m_{\boldsymbol{R}}(x) d m_{\Delta}(d) \\
& =\hat{f}((r, k)) \\
& =0 .
\end{aligned}
$$

Hence the uniqueness theorem implies

$$
\int_{\boldsymbol{R}} e^{-i x r} f(x, d) d m_{\boldsymbol{R}}(x)=0
$$

for each $d \varepsilon \Delta$ because $\Delta$ is discrete. Thus we have $f(\bullet, d) \varepsilon H^{1}(\boldsymbol{R})$ for each $d \varepsilon \Delta$. Conversely, suppose that $f \varepsilon L^{1}(\boldsymbol{R} \times \Delta)$ and $f(\cdot, d) \varepsilon H^{1}(\boldsymbol{R})$ for each $d \varepsilon \Delta$. Then $(f(\bullet, d))^{\wedge}(0)=0$ because $(f(\bullet, d)) \wedge$ is continuous on $\boldsymbol{R}$. Let $(r, k)$ \& $P$. From the form of $P$, we have $r \leqq 0$. Thus

$$
\begin{aligned}
& \int_{\boldsymbol{R} \times \Delta}(-(x, d),(r, k)) f(x, d) d m_{\boldsymbol{R} \times \Delta}(x, d) \\
& =\int_{\Delta}(-d, k) \int_{\boldsymbol{R}} e^{-i x r} f(x, d) d m_{\boldsymbol{R}}(x) d m_{\Delta}(d) \\
& =\int_{\Delta}(-d, k) \cdot 0 d m_{\Delta}(d) \\
& =0 .
\end{aligned}
$$


Hence we have $f \varepsilon H_{P}^{1}(\boldsymbol{R} \times \Delta)$. This proves the claim. By the claim, we can easily see that

$$
H_{P}^{\infty}(\boldsymbol{R} \times \Delta)=\left\{g \varepsilon L^{\infty}(\boldsymbol{R} \times \Delta): g(\cdot, d) \varepsilon H^{\infty}(\boldsymbol{R}) \text { for each } d \varepsilon \Delta\right\} .
$$

Once we obtain these, we can proceed in the same methods as Yamaguchi's ([16, Theorem 21]).

We next consider the case where $G$ is isomorphic to $\boldsymbol{T} \times \Delta$. By Remark 2, we may suppose

$$
P=\{(n, k) \varepsilon \boldsymbol{Z} \times K: n>0 \text { and } k \varepsilon K\} \cup(P \cap(\{0\} \times K)) .
$$

Since $P$ is dense in $\{0\} \times K$ (cf. [8, Theorem (9.16)]), we can easily verify that $P \supset\{0\} \times K$ if $P^{\circ} \cap(\{0\} \times K) \neq \phi$ and $(\{0\} \times K) \mid P$ is dense in $\{0\} \times K$ if $P^{\circ} \cap(\{0\} \times K)=\phi$, where $P^{\circ}$ denotes the interior of $P$. By using these facts and the above arguments for $G=\boldsymbol{R} \times \Delta$, it is easy to see that

$$
H_{P}^{1}(\boldsymbol{T} \times \Delta)=\left\{f \varepsilon L^{1}(\boldsymbol{T} \times \Delta): f(\bullet, d) \varepsilon H^{1}(\boldsymbol{T}) \text { for each } d \varepsilon \Delta\right\}
$$

or

$$
H_{P}^{1}(\boldsymbol{T} \times \Delta)=\left\{f \varepsilon L^{1}(\boldsymbol{T} \times \Delta): f(\cdot, d) \varepsilon H_{0}^{1}(\boldsymbol{T}) \text { for each } d \varepsilon \Delta\right\},
$$

where $H_{0}^{1}(\boldsymbol{T})=\left\{f \varepsilon H^{1}(\boldsymbol{T}): \hat{f}(0)=0\right\}$. Thus we have

$$
H_{P}^{\infty}(\boldsymbol{T} \times \Delta)=\left\{g \varepsilon L^{\infty}(\boldsymbol{T} \times \Delta): g(\cdot, d) \varepsilon H_{0}^{\infty}(\boldsymbol{T}) \text { for each } d \varepsilon \Delta\right\}
$$

or

$$
H_{P}^{\infty}(\boldsymbol{T} \times \Delta)=\left\{g \varepsilon L^{\infty}(\boldsymbol{T} \times \Delta): g(\bullet, d) \varepsilon H^{\infty}(\boldsymbol{T}) \text { for each } d \varepsilon \Delta\right\} .
$$

Hence we can proceed in the same methods as Yamaguchi's ([16, Theorem 20]).

(iv) $\Longleftrightarrow(\mathrm{v})$ : Note that $P$ has the FMR property if and only if $-P$ has the FMR property. Thus we see that $M_{P}(G) \subset L^{1}(G)$ implies $M_{P^{c}}(G) \subset L^{1}(G)$. Conversely, suppose $M_{P^{c}}(G) \subset L^{1}(G)$ and $\mu \varepsilon M_{(-P)}(G)$ and fix $\gamma_{0}$ \& $-P$. Then we have $P+\gamma_{0} \subset(-P)^{c}$. Hence

$$
\left(\bar{\gamma}_{0} \mu\right) \hat{(}(\gamma)=\hat{\mu}\left(\gamma+\gamma_{0}\right)=0
$$

for all $\gamma \varepsilon P$, and so $\bar{\gamma}_{0} \mu \varepsilon M_{P^{c}}(G)$, where $\bar{\gamma}_{0}$ denotes the complex conjugate of $\gamma_{0}$. By our assumption, $\bar{\gamma}_{0} \mu \varepsilon L^{1}(G)$ and therefore we have $\mu \varepsilon L^{1}(G)$.

REMARK 3. If $G$ is a locally compact Abelian group with ordered dual group $\hat{G}$ and if $P$ is an order in $\hat{G}$ which is not dense in $\hat{G}$, then the implications " (iii) $\Rightarrow(\mathrm{vi})$ " and " (iv) $\Rightarrow(\mathrm{vi})$ " can be easily proved by using only Lemma 4 and Theorem 1 in [10]. 
The proof of Theorem 3 in [18] is incomplete. But the following is true :

Theorem ([18, Corollary]). Let $p_{1}$ and $p_{2}$ be numbers in the closed interval [1,2]. Suppose $\mu \varepsilon M\left(\boldsymbol{R}^{N}\right)(N \geq 2)$ satisfies the following two conditions ;

(a) there exists a function $f \in L^{p_{1}}\left(\boldsymbol{R}^{N}\right)$ such that

$$
\hat{\mu}(t)=\hat{f}(t) m_{\boldsymbol{R}^{N}}-a . \text { e. } t \varepsilon\left\{t=\left(t_{j}\right) \varepsilon \boldsymbol{R}^{N}: t_{1} \geq 0\right\},
$$

and

(b) for each $t_{1}<0$,

$$
\hat{\mu}\left(t_{1}, u\right)=\hat{f}_{t_{1}}(u) m_{\boldsymbol{R}^{v}}-a . \text { e. } u \in \boldsymbol{R}^{N-1}
$$

for some $f_{t_{1}} \varepsilon L^{p_{2}}\left(\boldsymbol{R}^{N-1}\right)$.

Then $\mu$ is absolutely continuous with respect to the Lebesgue measure $m_{\boldsymbol{R}^{\mathrm{N}}}$ on $\boldsymbol{R}^{N}$.

Proof. Let $\mu$ be a measure satisfying our assumption. By the condition (a) and [2, Main Theorem], there exists a function $h$ in $L^{1}\left(\boldsymbol{R}^{N}\right)$ such that

$$
\hat{\mu}(t)=\hat{h}(t) m_{\boldsymbol{R}^{N}}-a . \text { e. } t \varepsilon\left\{t=\left(t_{j}\right) \varepsilon \boldsymbol{R}^{N}: t_{1}>0\right\} .
$$

Since $\hat{\mu}$ and $\hat{h}$ are continuous on $\boldsymbol{R}^{N}$ and the subset $\left\{t=\left(t_{j}\right) \varepsilon \boldsymbol{R}^{N}: t_{1}>0\right\}$ is open, we have

$$
\hat{\mu}(t)=\hat{h}(t) \text { on }\left\{t=\left(t_{j}\right) \varepsilon \boldsymbol{R}^{N}: t_{1}>0\right\}
$$

and hence on $\left\{t=\left(t_{j}\right) \varepsilon \quad \boldsymbol{R}^{N}: t_{1} \geq 0\right\}$. By the condition (b) and [8, Theorem 31.33], $\hat{\mu}\left(t_{1}, \bullet\right) \varepsilon\left(L^{1}\left(\boldsymbol{R}^{N-1}\right)\right)^{\wedge}$ for each $t_{1}<0$. Since $\hat{h}\left(t_{1}, \bullet\right) \varepsilon$ $\left(L^{1}\left(\boldsymbol{R}^{N-1}\right)\right)^{\wedge}$ for each $t_{1} \varepsilon \boldsymbol{R}$, we have

$$
\hat{\mu}\left(t_{1}, \cdot\right)-\hat{h}\left(t_{1}, \cdot\right) \varepsilon\left(L^{1}\left(\boldsymbol{R}^{N-1}\right)\right) \hat{.}
$$

By [18, Theorem 1], the measure $(\mu-h)$ is absolutely continuous with respect to $m_{\boldsymbol{R}^{N}}$. Then, of course, $\mu$ is absolutely continuous with respect to $m_{\boldsymbol{R}^{*}}$.

Acknowledgement. The author wound like to thank Professors S. Koshi and H. Yamaguchi for many helpful discussions and comments, and the referee for his helpful advice. 


\section{REFERENCES}

[1] K. dELEEUW and I. GLICKSBERG: Quasi-invariance and analyticity of measures on compact groups, Acta Math. 109 (1963), 179-205.

[ 2 ] R. Doss : On measures with small transforms, Pacific J. Math. 26 (1968), 257-263.

[ 3 ] I. GLICKSBERG : Spectra of invariant uniform and transform algebras, Trans. Amer. Math. Soc. 277 (1983), 381-396.

[ 4 ] I. GLICKSBERG : The strong conclusion of the F. and M. Riesz theorem on groups, Trans. Amer. Math. Soc. 285 (1984), 235-240.

[5] H. Helson and D. LOWDEnSlager: Prediction theory and Fourier series in several variables, Acta Math. 99 (1958), 165-202.

[ 6 ] E. HEWITT and S. KOSHI: Orderings in locally compact Abelian groups and the theorem of F. and M. Riesz, Math. Proc. Cambridge Phil. Soc. 93 (1983), 441-457.

[ 7 ] E. HewitT, S. Koshi, and Y. TAKAhASHI: The F. and M. Riesz theorem revisited, (to appear in Math. Scand.)

[ 8 ] E. HEwiTT and K. A. Ross : Abstract Harmonic Analysis, I and II. Springer-Verlag, Berlin-Heidelberg-Now York, 1963.

[ 9 ] S. KOSHI and Y. TAKAHASHI: Generating subsemigroups, orders, and a theorem of Glicksberg, (submitted to Hokkaido Math. J.)

[10] S. Koshi and H. YAmaGUCHI: The F. and M. Riesz theorem and group structures, Hokkaido Math. J. 8 (1979), 294-299.

[11] W. RUDIN : Fourier Analysis on Groups, Interscience, New York, 1962.

[12] W. RUdiN : Spaces of type $H^{\infty}+C$, Ann. Inst. Fourier (Grenoble). 25 (1975), 99-125.

[13] D. SARASON : Generalized interpolation in $H^{\infty}$, Trans. Amer. Math. Soc. 127 (1967), 179-203.

[14] D. SARASON : Functions of vanishing mean oscillation, Trans. Amer. Math. Soc. 207 (1975), 391-405.

[15] Y. TAKAHASHI and H. YAMAGUCHI: On measures which are continuous by certain translation, Hokkaido Math. J. 13 (1984), 109-117.

[16] H. YAMAGUCHI : On certain function spaces and group structures, Bull. Austral. Math. Soc. 19 (1978), 217-244.

[17] H. Yamaguchi : A property of some Fourier-Stieltjes transforms, Pacific J. Math. 108 (1983), 243-256.

[18] Y. TAKAhaShi : On a theorem of S. Saeki, Hokkaido Math. J. 15 (1986), 157-161.

Department of Mathematics

Hokkaido University 